\title{
Prevalence of abomasal lesions in Danish Holstein cows at the time of slaughter
}

\author{
S. L. Munch, ${ }^{1 *}$ S. S. Nielsen, ${ }^{2}$ M. A. Krogh, ${ }^{3}$ and N. Capion ${ }^{1}$ \\ ${ }^{1}$ Department of Veterinary Clinical Sciences, University of Copenhagen, Højbakkegård Alle 5A, DK-2630 Taastrup, Denmark \\ ${ }^{2}$ Department of Veterinary and Animal Sciences, University of Copenhagen, Grønnegårdsvej 8, DK-1870 Frederiksberg, Denmark \\ ${ }^{3}$ Department of Animal Science, Aarhus University, Blichers Alle 20, DK-8830 Tjele, Denmark
}

\section{ABSTRACT}

Abomasal lesions in cattle are challenging to diagnose because the clinical signs are often subtle and nonspecific. An increasing number of studies suggests that abomasal lesions are commonly found in cattle of all ages, but the number of recent prevalence studies in dairy cows is limited. The main objective of this study was to estimate the prevalence of abomasal lesions in a population of Danish Holstein dairy cattle in Denmark. An abattoir survey of 1,327 dairy cows apparently suitable for slaughter was performed in 2016 and 2017. Abomasal lesions are usually classified using a standardized protocol with the following types: abomasal ulcer type I: erosions and nonperforating ulcers; type II: ulcers with arterial bleeding; type III: perforating ulcer with localized peritonitis; and type IV: perforating ulcer with diffuse peritonitis. A further subdivision of the nonperforating abomasal ulcers type I were classified as subtype Ia: erosions; subtype Ib: small ulcers with localized hemorrhage; subtype Ic: ulcers with a crater-like appearance; and subtype Id: retention of the mucosa due to tissue loss with either radial wrinkles converging at a central point or perforations of the spiral folds. Type I abomasal lesions were found in $84 \%$ of the examined cows. No ulcers with arterial bleeding (type II) or perforating ulcers with diffuse peritonitis (type IV) were found, but one perforating abomasal ulcer (type III) was observed. The total number of lesions found was 7,418 and when the lesion subtypes were evaluated individually the majority of subtypes Ia and Ic were found in the pyloric area (85 and 94\%, respectively), whereas lesion subtypes $\mathrm{Ib}$ and Id dominated the fundus/corpus area (71 and $67 \%$, respectively). When considering lesion subtypes

Received September 27, 2018.

Accepted January 23, 2019.

*Corresponding author: slm@sund.ku.dk according to parity, there was a high prevalence of subtype $\mathrm{Ib}$ across the 3 groups of parity $(51,58$, and $55 \%$ for parity 1,2 , and $\geq 3$, respectively). The prevalence of lesion subtype Id seemed to increase with increasing parity, and the prevalence of lesion subtype Ia seemed to decrease with increasing parity. The prevalence of abomasal lesions was higher than that shown in similar studies of cattle at the time of slaughter. However, the expectedly most painful cases were rare, with only one perforating and no arterial bleeding ulcers found. The etiology of the different subtype I lesions is still unknown, and further investigations are needed to establish the possible influence these lesions might have on welfare and production.

Key words: abomasal ulcer, abattoir investigation, dairy cow, prevalence

\section{INTRODUCTION}

Abomasal lesions in dairy cows may cause heavily bleeding or perforating ulcers, which can result in anemia, peritonitis, and death (Palmer and Whitlock, 1984; Smith et al., 1986). However, the majority of abomasal lesions have a less obvious clinical presentation, and the prevalence is rarely estimated (Whitlock, 1980).

Abomasal lesions are divided into 4 types according to the distinct variations in clinical signs between the types (Whitlock, 1980). Abomasal lesion type I and II are nonperforating and type III and IV are perforating ulcers. Type I includes erosions and ulcers, and type II includes ulcers with arterial bleeding from the left or right gastroepiploic artery. Type III are ulcers with acute, circumscribed peritonitis, and type IV are ulcerations with diffuse peritonitis (Whitlock, 1980). The type I category of erosions and nonperforating ulcers contains both acute and chronic lesions. The differences in macroscopic morphology of acute and chronic type I lesions encouraged Braun et al. (1991) to make a subclassification of the type I lesions: subtype Ia: erosions with minimal mucosal defects; subtype Ib: sharply defined ulcers with a punched-out appearance and lo- 
cal mucosal hemorrhage; subtype Ic: crater-like ulcers with a superficial coating of detritus (e.g., fibrin or inflammatory products); and subtype Id: radial wrinkles originating from different directions and converging at a central point (star-shaped scars) or holes through the spiral folds (Braun et al., 1991).

Ante-mortem diagnosis of type I abomasal lesions in cows is challenging. The clinical signs are often absent or subtle, and include general signs of gastro-intestinal discomfort such as nonspecific abdominal pain, anorexia, dehydration, or hypomotility of the rumen (Smith et al., 1983; Braun et al., 1991). Nonspecific abdominal pain can be recognized by the withers pinch or the bar test (Jackson and Cockcroft, 2002).

The prevalence of abomasal lesions at the time of slaughter has been reported in the range of 11 to $49 \%$ in cows (Braun et al., 1991; Mesarič, 2005; Hund et al., 2016). Braun et al. (1991) examined 912 randomly selected cows of the Swiss Braunvieh, Swiss Holstein, and Simmental breeds. They reported an overall abomasal lesion prevalence of $21 \%$. Hund et al. (2016) reported abomasal lesions in a population of 33 predominantly Simmental cows, of which $49 \%$ had abomasal lesion subtypes Ia, Ib, and Ic. Mesarič (2005) examined the abomasa of 126 cows and found the prevalence of ulcers was $11 \%$, whereas $17 \%$ had no changes in the abomasum and $71 \%$ had other unspecified changes in the abomasum. An older study on abomasal lesions at slaughter in an apparently healthy Danish cattle population reported that $33 \%$ of cows and bulls (age $\geq 2 \mathrm{yr}$ ) had erosions, active ulcers, or both in the abomasum (Hemmingsen, 1966). This study involved examinations of 1,712 abomasa from mostly Danish Red cattle, the majority of which were female $(\mathrm{n}=1,153)$ and considered to be adult, with an age $\geq 2$ yr $(n=910)$. The type and subtype of the lesions found in the abomasum by Hemmingsen (1966) were mostly comparable to those categorized by Braun et al. (1991) as abomasal lesion subtypes Ia, Ib, and Ic.

Danish dairy production has changed considerably since the studies from the 1960s [e.g., the average yearly milk yield for Danish Holstein cows has increased from less than $5,000 \mathrm{~kg}$ in 1960 to more than $10,000 \mathrm{~kg}$ per cow in 2015 (SEGES, 2015)]. To our knowledge, no recent prevalence studies have investigated animals in intensive production systems.

The primary objective of this study was to estimate the prevalence of abomasal lesions in Danish Holstein dairy cows at the time of slaughter. Our secondary objective was to estimate the distribution of different subtypes of type I lesions found in the abomasa of Danish Holstein dairy cows at the time of slaughter.

\section{MATERIALS AND METHODS}

\section{Study Population}

The sample consisted of Danish Holstein cows that farmers found suitable for transport and slaughter as a part of the normal culling procedure in the dairy production systems. Cows that were rejected for human consumption in the slaughter process were excluded from the study. Animals were observed and selected at the beginning of the slaughter chain. Individual cows were identified after sampling at the abattoir via the slaughter ID, which was obtained for every cow. Animals were considered to be Danish Holstein cows if they had a black pied coat and adult udder conformation. If the animal was not identified as a Danish Holstein in a public database (https://chr.fvst.dk), sample results were subsequently excluded from the study.

\section{Study Design}

The study was performed as an observational crosssectional study at 2 different abattoirs (A and B) in Denmark. The abattoirs were chosen for accessibility and practical reasons. It was important that the abattoir facilities allowed one person to collect the study material with ample precision and quality without being a nuisance to the regular staff at the abattoirs. The abomasa of selected cows were examined for the presence of lesions and other pathoanatomical abnormalities. From abattoir A, 29 abomasa were sampled from January to March 2016. From abattoir B, 1,600 abomasa were sampled over 3 sampling periods between March 2016 and June 2017. In total, 1,629 abomasa were collected and examined by the same observer ( $\mathrm{S}$. L. Munch).

After evisceration of the carcass, the intestines were further parted. When the abomasum was severed from the rest of the gastro-intestinal tract, it was opened along the greater curvature. Abomasal content was removed and, if necessary, washed with water. All macroscopic lesions were registered according to anatomical site (specified as zone 1-3) and severity (no lesions, subtype Ia-Id lesions, or type II-IV lesions). Lesions were categorized according to Whitlock (1980) and Braun et al. (1991). Due to a lack of consensus of the anatomical definitions in the abomasum, we divided the abomasum in 3 distinct zones based on the macroscopic appearance (Figure 1). Zone 1 starts at the omasoabomasal orifice and stretches to the end of the abomasal folds where the mucosa changes from uniform and smooth to a rougher appearance. This is defined as either the fundus or the corpus or a mix of 


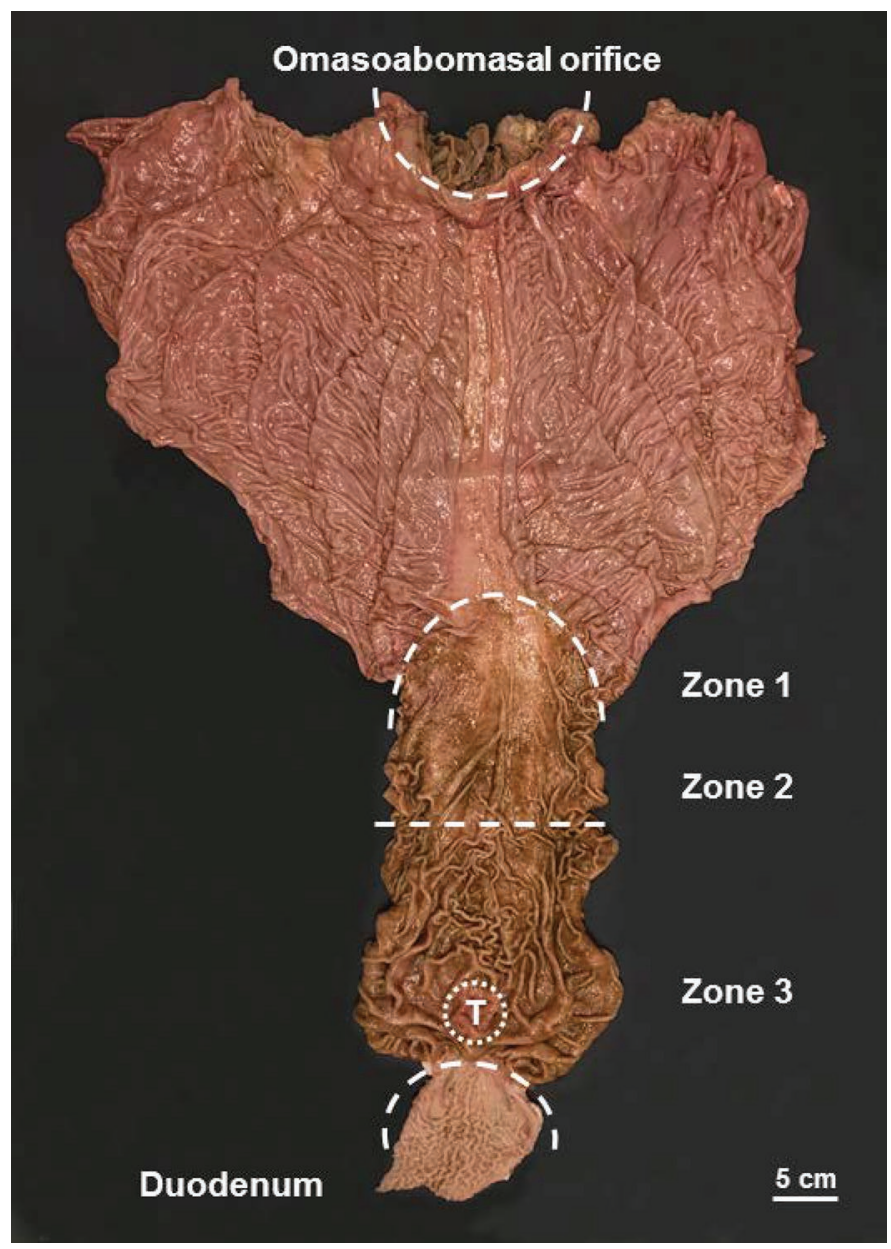

Figure 1. Illustration of an abomasum. The abomasum has been opened along the greater curvature from the omasoabomasal orifice to the duodenum. We have divided the abomasum into zones 1 to 3 according to the macroscopic presentation. Zone 1 is the most oral part of the abomasum and begins at the omasoabomasal orifice. It extends to where the large spiral folds end. Zone 2 is a more flat area without much mucosal folding. Zone 2 is located between the large abomasal folds in zone 1 and the smaller folds in zone 3 . Zone 3 has more mucosal folds than zone 2 and is located in the most aboral part of the abomasum. The torus pyloricus $(\mathrm{T})$ is located in zone 3 just before the duodenum.

those in different anatomical veterinary textbooks (e.g., Dyce et al., 2002; Eurell and Frappier, 2006; Schaller, 2007). Zone 2 is the middle part between the abomasal folds and the much smaller mucosal folds in the most aboral part of abomasum. Zone 3 is the most aboral part of the abomasum just before the duodenum and is distinguished from zone 2 by the presence many small folds in the mucosa. The torus pyloricus is also located here. Examination of the abomasum began in zone 1 by inspecting the spiral folds one by one from right to left, then zone 2 was examined for lesions, followed by the visual inspection of zone 3 . One hand was used to unfold the mucosa of the abomasum to level the surface while pictures of the lesions were taken using the other hand. Changes in the abomasal mucosa such as nodules, hyperplasia, or edema were also registered.

The pictures were assessed by the first author following sampling at the abattoirs. If a picture showed a small number of lesions, the largest lesion was registered first, followed by the second largest, and so on, ending with the smallest. If a picture showed many lesions, the assessor started in one corner of the picture and measured the lesions systematically across the picture to ensure that all lesions were registered. In an effort to make our results comparable with findings in previous studies, the lesions were categorized according to the main (i.e., most frequent) lesion type in each abomasum, as described by Braun et al. (1991). If an equal number of lesion types were present in the abomasum, they were classified as follows: Abomasal lesion subtype Id $>$ Ic $>$ Ib $>$ Ia.

\section{RESULTS}

In total, 1,629 abomasal samples were collected at the abattoir and 1,327 Danish Holstein dairy cows met the inclusion criteria and were included in the study. Reasons for exclusion were non-Holstein breed $(\mathrm{n}=$ $265)$, Holstein heifers $(\mathrm{n}=30)$, Holstein cows from herds that had not delivered milk for the last year $(\mathrm{n}=$ $5)$, and Holstein bulls $(\mathrm{n}=2)$.

No type II or type IV lesions were observed, but one type III perforating abomasal ulcer communicating with an abscess on the serosa of the abomasum was observed. Type I lesions were found in 1,119 abomasa samples (84\%). The results of categorizing by main lesion subtypes I are shown in Table 1. Subtype Ic constituted the highest prevalence $(34 \%)$ of all the main lesion types. Main lesion subtypes Ia and Ib had similar prevalences at 25 and $21 \%$, respectively, and main lesion subtype Id had the lowest prevalence $(2.9 \%)$ of all examined abomasa.

Table 1. The distribution of abomasal lesion type I subtypes Ia-Id found in Danish Holstein dairy cattle at the time of slaughter ${ }^{1}$

\begin{tabular}{lcc}
\hline & $\begin{array}{c}\text { Percent of } \\
\text { examined abomasa } \\
(\mathrm{n}=1,326)\end{array}$ & $\begin{array}{c}\text { Percent of } \\
\text { abomasa with } \\
\text { lesions }(\mathrm{n}=1,119)\end{array}$ \\
\hline Ia & $25(23-28)$ & 30 \\
Ib & $21(19-24)$ & 25 \\
Ic & $34(32-37)$ & 41 \\
Id & $2.9(2.0-3.8)$ & 3.4 \\
Total & 84 & 100 \\
\hline
\end{tabular}

${ }^{1}$ The lesions are registered according to the most common lesion subtype found in the abomasum. Results are shown as proportions of the total number of animals, with a CI of $95 \%$. 
Table 2. The quantity of lesion subtypes Ia-Id by anatomical location in 1,327 Danish Holstein dairy cattle at the time of slaughter in 2016 and $2017^{1}$

\begin{tabular}{lccccc}
\hline Lesion subtype & $\begin{array}{c}\text { Zone } 1^{2} \\
(\%)\end{array}$ & $\begin{array}{c}\text { Zone } 2^{2} \\
(\%)\end{array}$ & $\begin{array}{c}\text { Zone } 3^{2} \\
(\%)\end{array}$ & $\begin{array}{c}\text { Total no. } \\
\text { of lesions }\end{array}$ & $\begin{array}{c}\text { Percent of cows } \\
\text { with lesions }\end{array}$ \\
\hline Ia & 7.5 & 7.6 & 85 & 1,342 & 43 \\
Ib & 71 & 8.4 & 20 & 4,089 & 32 \\
Ic & 1.2 & 5.2 & 94 & 1,774 & 51 \\
Id & 67 & 20 & 13 & 213 & 7.1 \\
Total population & 43 & 7.8 & 49 & 7,418 & 84 \\
\hline
\end{tabular}

${ }^{1}$ It should be noted that one animal can have multiple lesions in the same anatomical location, or multiple lesions in different anatomical locations, and that one animal can have more than one lesion subtype.

${ }^{2}$ Anatomical locations in the abomasum. Zone 1 is the most oral part of the abomasum with spiral folds. Zone 2 is a thicker mucosa than zone 1 , but with very few wrinkly folds located between the smooth mucosa with spiral folds of zone 1 and the thicker wrinkled mucosa of zone 3, which is the most aboral part of the abomasum. See also Figure 1 for a visual explanation.

Of the 1,120 abomasa with lesions, 598 (53\%) had 1 type of lesion, 395 (35\%) had 2 types of lesion, 119 (11\%) had 3 types of lesion, and $8(0.7 \%)$ abomasa had all 4 types at the time of slaughter.

Table 2 shows the distribution according to subtypes of lesion type I and macroscopic location (zone) of all 7,423 lesions found in the abomasa. There seemed to be an affinity of lesion subtype according to zone; the majority of abomasal lesion subtypes Ia (85\%) and Ic (94\%) were primarily found in zone 3 , whereas 71 and $67 \%$ of abomasal lesion subtypes Ib and Id, respectively, were found in zone 1 . Cows with lesion subtype Ia had an average of 2.3 lesions, those with subtype $\mathrm{Ib}$ had an average of 9.5 lesions, those with subtype Ic had an average of 2.6 lesions, and cows with subtype Id had an average of 2.4 lesions.

The distribution of lesion I subtypes according to parity is shown in Table 3. Included in the groups were 268 first-parity cows, 313 second-parity cows, and 539 third-or-more-parity cows. More than half of all lesions found in each parity $(1,2$, and $\geq 3)$ were subtype $\mathrm{Ib}$, ranging between $51 \%$ and $58 \%$. Lesion subtype Id was the smallest proportion of all lesion subtypes, but was relatively more frequent in animals with increasing parity. Lesion subtype Ia seemed to be most common in first-parity cows (with a prevalence of $24 \%$ ) compared with second- and third-or-more-parity cows (with prevalences of 18 and 15\%, respectively).
The combination of different lesion subtypes and their numbers are shown in Table 4. The majority of abomasa (63\%) had 1-5 lesions. However, $4.4 \%$ of animals had $\geq 21$ lesions in their abomasa, with a maximum of 243 lesions (subtype $\mathrm{Ib}$ ) found in 1 animal. All lesion type I subtypes were present in 8 of the cows, 5 cows had 6-20 lesions, and 3 cows had $\geq 21$ lesions.

A total of 1,034 abomasa (78\%) had hyperplasia or nodules in the mucosa. Scars not compatible with the description of the subtype Id $(\mathrm{n}=70)$ were found in 45 abomasa (3.4\%). The scars either had an elongated and almost mesh-like appearance or were very large circular structures in the most aboral part of the zone 3 , on or beside the torus pyloricus. The scars were primarily observed in the zone 3 or zone 1 . Edema was found in 11 of the abomasa $(0.8 \%)$ at the time of slaughter. During the time of the study, 3 animals $(0.2 \%)$ were registered having adherences with varying degrees of involvement of the abdomen.

\section{DISCUSSION}

We found one type III lesion and no type II or IV lesions. With these results we focused our attention to the subtypes of the type I lesions. We observed a prevalence of $84 \%$ of Holstein dairy cows with type I lesions. To our knowledge, this is the highest prevalence of lesions reported in dairy cattle. The study by Mesarič

Table 3. The quantity of lesion subtypes Ia-Id in the abomasum according to the parity of 1,327 Danish Holstein cows at the time of slaughter in 2016 and $2017^{1}$

\begin{tabular}{lcccccc}
\hline & \multicolumn{5}{c}{ Subtypes of lesion type I (\%) } & No. of \\
\cline { 2 - 5 } Parity & Ia & Ib & Ic & Id & & $\begin{array}{c}\text { Percent of cows } \\
\text { with lesions }\end{array}$ \\
\hline 1 & 24 & 51 & 24 & 1.4 & 1,577 & 81 \\
2 & 18 & 58 & 22 & 2.2 & 2,168 & 86 \\
$\geq 3$ & 15 & 55 & 25 & 4.4 & 3,673 & 85 \\
Total population & 18 & 55 & 24 & 3.1 & 7,418 & 84 \\
\hline
\end{tabular}

${ }^{1}$ It should be noted that one animal can have multiple lesions as well as multiple lesion subtypes simultaneously. 
(2005) could have reported a similar prevalence of abomasal lesions. However, they estimated the prevalence of ulcers [type I and type II using the definitions by Whitlock (1980)] to $11 \%$, but if their "erosions and abomasitis" are also considered as type I lesions, then prevalence was $82 \%$ and thereby very similar to our findings.

The presentation of results from different studies generally lacked consistency. Previous studies may have recorded less detail or the study populations could have had fewer lesions or lesion types due to breed, sex, or age when they are compared with our study population. Hemmingsen (1966) presented his lesion results in 3 categories: erosions, ulcers, and both erosions and ulcers, with a prevalence of $28,2.6$, and $2.4 \%$, respectively, for animals older than 24 mo. Aukema and Breukink (1974) had 3 categories of lesions: erosions, ulcers, and scars, with a prevalence of 6.2 and $7.2 \%$ of erosions in the fundus and pylorus, respectively. They also reported a prevalence of $1 \%$ for ulcers and $5.3 \%$ for abomasal scars. Braun et al. (1991) and Hund et al. (2016) organized their lesions by most frequent lesion type, thereby excluding the possibility of reporting more than one lesion type per abomasum. We also organized our results according to the main lesion subtype as shown in Table 1 for comparative purposes. However, Braun et al. (1991) did not describe how they categorized abomasa with an equal number of lesions of more than one lesion subtype. Therefore, we decided to categorize as the main lesion using the order Id $>$ Ic $>$ Ib $>$ Ia. However, we found the categorizing according to main lesion subtype did not show the same results as if the lesions were presented according to le- sion subtype. An example of this was lesion subtype Ib being the most common lesion type, constituting $55 \%$ of the total number of lesions found $(\mathrm{n}=4,089)$ as shown in Tables 2 and 3, yet it was only identified as the main lesion subtype in $21 \%$ of all abomasa and $25 \%$ of the abomasa with lesions (Table 1). One reason for this is that lesion subtype Ib often occurs in a large number (as shown in Table 4), both as a single lesion type, but also in combination with other lesion types. When only the main lesion subtype is considered, any other types that are present will be disregarded. We find the presentation by main lesion subtype troublesome and inadequate to describe the whole picture. The investigations made previously on abomasal lesions have divided the abomasum into different anatomical regions. There seems to be little consensus about how the anatomical division of the abomasum should be made. Hemmingsen (1966) divided his abomasum into pars fundica (fundus) and pars pylorica (pylorus); the fundus described by Hemmingsen (1966) corresponds to our zone 1 and our zones 2 and 3 correspond to his depiction of the pylorus. Braun et al. (1991) seemed to use the same split with division into fundus and pylorus of the abomasum as Hemmingsen (1966). However, they did not provide a detailed explanation of how they distinguished anatomically between the fundus and the pylorus. Hund et al. (2016) used a different anatomical division of the abomasum; they did not provide a detailed description of how they divided the abomasum either. Nonetheless, we believe their corpus corresponded to our zone 1 and their pylorus corresponded to our zone 2 and 3. Mesarič (2005) gave no description of findings according to anatomical location.

Table 4. Cross tabulation of the frequency of each lesion subtype and lesion subtype combination found in 1,327 Danish Holstein cows at the time of slaughter in 2016 and 2017

\begin{tabular}{|c|c|c|c|c|}
\hline \multirow{2}{*}{$\begin{array}{l}\text { Lesion subtype or } \\
\text { combinations }\end{array}$} & \multicolumn{3}{|c|}{ No. of lesions per abomasum } & \multirow{2}{*}{$\begin{array}{l}\text { Percent of } \\
\text { cows }\end{array}$} \\
\hline & 1-5 Lesions & 6-20 Lesions & $\geq 21$ Lesions & \\
\hline None & 0 & 0 & 0 & 16 \\
\hline Ia & 221 & 7 & 1 & 17 \\
\hline $\mathrm{Ib}$ & 70 & 21 & 11 & 7.7 \\
\hline Ic & 234 & 24 & 0 & 19 \\
\hline Id & 8 & 1 & 0 & 0.7 \\
\hline $\mathrm{Ia}+\mathrm{Ib}$ & 56 & 21 & 5 & 6.2 \\
\hline $\mathrm{Ia}+\mathrm{Ic}$ & 115 & 30 & 1 & 11 \\
\hline $\mathrm{Ia}+\mathrm{Id}$ & 5 & 4 & 0 & 0.7 \\
\hline $\mathrm{Ib}+\mathrm{Ic}$ & 64 & 48 & 17 & 9.7 \\
\hline $\mathrm{Ib}+\mathrm{Id}$ & 4 & 1 & 0 & 0.4 \\
\hline $\mathrm{Ic}+\mathrm{Id}$ & 17 & 5 & 1 & 1.7 \\
\hline $\mathrm{Ia}+\mathrm{Ib}+\mathrm{Ic}$ & 26 & 40 & 12 & 5.9 \\
\hline $\mathrm{Ia}+\mathrm{Ib}+\mathrm{Id}$ & 2 & 3 & 1 & 0.5 \\
\hline $\mathrm{Ia}+\mathrm{Ic}+\mathrm{Id}$ & 11 & 5 & 1 & 1.3 \\
\hline $\mathrm{Ib}+\mathrm{Ic}+\mathrm{Id}$ & 3 & 9 & 6 & 1.4 \\
\hline $\mathrm{Ia}+\mathrm{Ib}+\mathrm{Ic}+\mathrm{Id}$ & 0 & 5 & 3 & 0.6 \\
\hline Id + III & 1 & 0 & 0 & 0.1 \\
\hline Total & 837 & 224 & 59 & 100 \\
\hline
\end{tabular}


We currently do not have tools to rank lesion types according to their effect on the welfare or production of the cow. However, from a pathophysiological point of view, acute lesions are likely to cause more pain than chronic lesions. Therefore, the acute subtypes Ia and Ib could be regarded with a higher effect on pain perception of the cow compared with cows with more chronic lesion subtypes Ic and Id. Nevertheless, if pain is not the primary focus, it could be argued that the chronic lesions give rise to the highest effect on the cow due to a longer compromised physical barrier between the abomasal mucosa and the blood stream. This breach in integrity causes the gastric juices and ingesta to have a direct contact with the blood stream, which can cause other complications regarding illness. Some of the acute lesions can also cause a breach in the physical barrier of the abomasum. We have no evidence to describe when the lesions develop and it is hypothesized that it even could be during transportation to the abattoir.

We found a higher proportion of cows with type I abomasal lesions sent for slaughter than previously reported. Braun et al. (1991) reported an overall type I abomasal lesion prevalence of $21 \%$, whereas Hund et al. (2016) reported an overall prevalence of $49 \%$ of adult cows with type I abomasal lesions, compared with the $84 \%$ observed here. The cows reported in our study were Holstein dairy cows from intensive production systems, whereas this is not necessarily the case in other studies. However, the distribution of concurrent lesion types may be more similar between studies. Of the 187 abomasa with lesions reported by Braun et al. (1991), 43 and $51 \%$ had 1 or 2 lesion types, respectively, whereas we found 53 and $35 \%$ with 1 or 2 lesion types. In addition, Braun et al. (1991) found 4.8\% with 3 lesion types, whereas we found $11 \%$, and the prevalence with 4 lesion types was reported by Braun et al. (1991) at $0.2 \%$, whereas we found $0.6 \%$. Overall, we observed a higher percentage of abomasa with 1,3 , or 4 abomasal lesion types than Braun et al. (1991), but the relative distribution appeared to be similar. Another explanation of the higher prevalence of abomasal lesions than previously reported could be due to the difference in breed and husbandry practices between countries. Nonetheless, the confounding of breed or husbandry practices does not make the potential problem with a high prevalence of abomasal lesions in dairy cows less important. The relative distribution could furthermore be used to target specific conditions (e.g., chronic versus acute lesion subtypes) because they may have a different effect on the cows.

Our overall distribution of lesions (Table 2) shows the same affinity for the different anatomic compartments as Braun et al. (1991). They reported that $75 \%$ of the lesion subtype Ia and $71 \%$ of lesion subtype Ic were found in the pylorus (i.e., zone 3). Furthermore, they reported that $71 \%$ of lesion subtype Ib and $69 \%$ of lesion subtype Id were found in the fundus (i.e., zone 1). Our results also show that lesion subtypes Ia and Ic have a relatively high affinity for zone 3 of the abomasum. We found $85 \%$ of lesion subtype Ia and $94 \%$ of lesion subtype Ic in zone 3. Likewise we found lesion subtypes Ib and Id had a high affinity for zone 1, where we found $71 \%$ of all Ib lesions and $67 \%$ of all subtype Id lesions. One of the possible explanations for why the lesion subtype Id was found with a high prevalence at zone 1 could be caused by one of the definitions of subtype Id. Because the definition includes holes in the spiral folds, this limitation of location causes a maybe false high prevalence of the lesion type at a specific area with the corresponding false low prevalence of the lesion type at the other possible areas. The subtype Id lesions described above were primarily found in the fundus compared with $20 \%$ of lesion were found in zone 2 and $13 \%$ in zone 3, as shown in Table 2. Also the location of the lesion subtypes according to anatomy could be explained by a possible difference in etiology of the different lesion subtypes.

The distribution of lesions according to parity also showed an interesting trend. The prevalence of lesion subtype Ic was found to be rather similar for the 3 groups of parity (Table 3). The prevalence of lesion subtype Id increased with increasing parity, whereas the prevalence of subtype Ia decreased with increasing parity. It could be speculated that some of the acute lesions found in the younger cows with time become chronic lesions. However, the majority of subtypes Ia and Id lesions were not found in the same anatomical areas of the abomasum, whereas Ia may become Ic, as they are primarily located in the same areas.

The prevalence we found in the slaughtered study population could be artificially high when compared with a population of Holstein dairy cows at farms. The cows included in this study could be sent for slaughter because they were affected by conditions resulting from either primary abomasal lesions or causing secondary abomasal lesions. Danish dairy cows are slaughtered for a variety of reasons (e.g., mastitis, metabolic disorders, and lameness). Some of the reasons mentioned above could be a source of pain or lead to reduced intake of food and water, which could be a cause for secondary abomasal lesions to form.

The absence of type IV ulcers could be due to the nature of the ulcers causing generalized peritonitis, and these cows are relatively unlikely to be suitable for neither transport (except for hospitalization) nor slaughter. This is why animals with lesion types II-IV typically are described in studies of hospitalized animals or studies of cattle that have died on their own 
(Smith et al., 1983; Palmer and Whitlock, 1984). The 3 animals observed with adherences in the abdomen could have been candidates for finding type IV ulcers at the abattoirs. However, none of the cows had any signs of perforating abomasal ulcers.

\section{CONCLUSIONS}

We found that $84 \%$ of apparently healthy Danish Holstein cattle had type I abomasal lesions at the time of slaughter. One type III abomasal ulcer was found, but no ulcer types II or IV were encountered. Additionally, we established that almost half $(47 \%)$ of the 1,120 abomasa with lesions had more than one of the abomasal lesion subtypes I present concurrently. Further investigations are needed to provide information about the age of the lesions and whether there is a specific point in time when cattle are particularly susceptible to lesion formation. In addition, further research is needed on the possible negative effects caused by the lesions in cattle.

\section{ACKNOWLEDGMENTS}

The authors acknowledge the staff at abattoir A and $\mathrm{B}$ for allowing the first author to work beside them while conducting this study and Jørgen S. Agerholm (University of Copenhagen) for photographing and editing the photo of the abomasum. This study was funded by the Danish Milk Levy Foundation (Aarhus). The authors have no conflicts of interest to declare. The study material was collected subsequent the slaughter- ing process of the cows and did not require an ethical approval from a governmental institution.

\section{REFERENCES}

Aukema, J. J., and H. J. Breukink. 1974. Abomasal ulcer in adult cattle with fatal haemorrhage. Cornell Vet. 64:303-317.

Braun, U., R. Eicher, and F. Ehrensperger. 1991. Type 1 abomasal ulcers in dairy cattle. Zentralbl. Veterinarmed. A 38:357-366.

Dyce, K. M., W. O. Sack, and C. J. G. Wensing. 2002. The abdomen of the ruminant. Pages 678-679 in Textbook of Veterinary Anatomy. Saunders, Philadelphia, PA.

Eurell, J. A., and B. L. Frappier. 2006. Digestive system. Pages 186194 in Dellmann's Textbook of Veterinary Histology. Blackwell Publishing Ltd., Oxford, UK.

Hemmingsen, I. 1966. Erosiones et ulcera abomasi bovis. Nord. Vet. Med. 18:354-365.

Hund, A., T. Beer, and T. Wittek. 2016. Abomasal ulcers in slaughtered cattle in Austria. Tierarztl. Prax. Ausg. G Grosstiere Nutztiere 44:279-285.

Jackson, P., and P. Cockcroft. 2002. Clinical examination of the gastrointestinal system. Pages 90-91 in Clinical Examination of Farm Animals. Blackwell Publishing Company, Iowa State Press, Ames.

Mesarič, M. 2005. Role of serum pepsinogen in detecting cows with abomasal ulcer. Vet. Arh. 75:111-118.

Palmer, J. E., and R. H. Whitlock. 1984. Perforated abomasal ulcers in adult dairy cows. J. Am. Vet. Med. Assoc. 184:171-174.

Schaller, O. 2007. Splanhnologia. Pages 158-159 in Illustrated Veterinary Anatomical Nomenclature. Enke Verlag, Stuttgart, Germany.

SEGES. 2015. Mælkeydelsens udvikling målt i ydelseskontrollen. in RYK - Ydelseskontrol. SEGES. Accessed Aug. 16, 2018. https://www.landbrugsinfo.dk/Kvaeg/RYK/Sider/ Maelkeydelsensudviklingmaaltiydelseskontrollen.aspx.

Smith, D. F., L. Munson, and H. N. Erb. 1983. Abomasal ulcer disease in adult dairy cattle. Cornell Vet. 73:213-224.

Smith, D. F., L. Munson, and H. N. Erb. 1986. Predictive values for clinical signs of abomasal ulcer disease in adult dairy-cattle. Prev. Vet. Med. 3:573-580.

Whitlock, R. H. 1980. Part II. Bovine Stomach Disease. Pages 396-433 in Veterinary Gastroenterology. 1st ed. N. V. Anderson, ed. Lea \& Febiger, Great Britain, by Bailiere Tindall, London, UK. 